\title{
Cyrius: accurate CYP2D6 genotyping using whole-genome sequencing data
}

\author{
Xiao Chen $\mathbb{1}^{1} \cdot$ Fei Shen ${ }^{1} \cdot$ Nina Gonzaludo ${ }^{1} \cdot$ Alka Malhotra $^{1} \cdot$ Cande Rogert $^{1} \cdot$ Ryan J. Taft ${ }^{1} \cdot$ David R. Bentley $^{2}$. \\ Michael A. Eberle $\mathbb{B}^{1}$
}

Received: 4 August 2020 / Revised: 13 November 2020 / Accepted: 4 December 2020 / Published online: 18 January 2021

(c) The Author(s) 2021. This article is published with open access, corrected publication 2022

\begin{abstract}
Responsible for the metabolism of $\sim 21 \%$ of clinically used drugs, CYP2D6 is a critical component of personalized medicine initiatives. Genotyping $C Y P 2 D 6$ is challenging due to sequence similarity with its pseudogene paralog $C Y P 2 D 7$ and a high number and variety of common structural variants (SVs). Here we describe a novel bioinformatics method, Cyrius, that accurately genotypes CYP2D6 using whole-genome sequencing (WGS) data. We show that Cyrius has superior performance (96.5\% concordance with truth genotypes) compared to existing methods (84-86.8\%). After implementing the improvements identified from the comparison against the truth data, Cyrius's accuracy has since been improved to 99.3\% . Using Cyrius, we built a haplotype frequency database from 2504 ethnically diverse samples and estimate that SVcontaining star alleles are more frequent than previously reported. Cyrius will be an important tool to incorporate pharmacogenomics in WGS-based precision medicine initiatives.
\end{abstract}

\section{Introduction}

There is significant variation in the response of individuals to a large number of clinically prescribed drugs. A strong contributing factor to this variability in drug metabolism is the genetic composition of the drug-metabolizing enzymes, and thus genotyping pharmacogenes is important for personalized medicine [1]. Cytochrome P450 2D6 (CYP2D6) encodes one of the most important drug-metabolizing enzymes and is responsible for the metabolism of about $21 \%$ of clinically used drugs [2]. The CYP2D6 gene is highly polymorphic, with 131 star alleles defined by the Pharmacogene Variation (PharmVar) Consortium [3] (as of 7/15/2020). Star alleles [4] are CYP2D6 haplotypes defined by a combination of small variants (SNVs and indels) and

Supplementary information The online version of this article (https:// doi.org/10.1038/s41397-020-00205-5) contains supplementary material, which is available to authorized users.

Michael A. Eberle

meberle@illumina.com

Illumina Inc., 5200 Illumina Way, San Diego, CA, USA

2 Illumina Cambridge Ltd., Illumina Centre, 19 Granta Park, Great Abington, Cambridge, UK structural variants (SVs), and correspond to different levels of CYP2D6 enzymatic activity, i.e., poor, intermediate, normal, or ultrarapid metabolizer [5-7].

Genotyping CYP2D6 is challenged by common deletions and duplications of CYP2D6 and hybrids between CYP2D6 and its pseudogene paralog, CYP2D7 [4, 8, 9], which shares $94 \%$ sequence similarity, including a few near-identical regions $[8,10]$. The interrogation of SVs improves the accuracy of CYP2D6 phenotype prediction [11]. Traditionally, CYP2D6 genotyping is done in low or medium throughput with array-based platforms, such as the PharmacoScan, or polymerase chain reaction (PCR) based methods such as TaqMan assays, ddPCR, and long-range PCR. These assays differ in the number of star alleles (variants) they interrogate, leading to variability in genotyping results across assays [8, 12, 13]. To detect SVs, these assays or test platforms may need to be complemented with CNV assays that may also be limited to detection of just a subset of the known CNVs [4, 9].

With recent advances in next-generation sequencing, it is now possible to profile the entire genome at highthroughput and in a clinically-relevant timeframe. Driven by these advances, many countries are undertaking large scale population sequencing projects [14-16] wherein pharmacogenomics testing will greatly increase the clinical utility of these efforts. There exist a few bioinformatics 
tools for genotyping CYP2D6 (Cypiripi [17], Astrolabe (formerly Constellation) [18], Aldy [19], and Stargazer $[20,21])$ that can be applied to targeted (PGRNseq [22]) and/or whole-genome sequencing (WGS) data. Among these, Cypiripi and Astrolabe were not designed to detect complex SVs and have been shown to have lower performance than the more recently developed methods $[19,23,24]$. The two most recent CYP2D6 callers, Aldy and Stargazer, work by detecting SVs based on sequencing coverage and calling star alleles based on the observed small variants and SVs. They rely on accurate read alignments, which may not be possible at many positions throughout the gene as the sequence is highly similar or even indistinguishable with CYP2D7. Relying on the initial read alignments may lead to ambiguous read coverage patterns or false positive/negative small variant calls. Another limitation of both Aldy and Stargazer is that, at the time this manuscript was written, neither method supports the GRCh38 genome build so studies using the latest genome build (GRCh38) will require a re-alignment to GRCh37 to use these tools.

Here we describe Cyrius, a novel WGS-specific CYP2D6 genotyping tool that overcomes the challenges with the homology between CYP2D6 and CYP2D7 and works for sequence data aligned to both GRCh38 and GRCh37. The availability of a panel of reference samples by the CDC Genetic Testing Reference Material Program (GeT-RM) $[12,25]$, where the consensus genotypes of major pharmacogenes are derived using multiple genotyping platforms, has enabled assessment of genotyping accuracy for newly developed methods. Furthermore, the recent availability of high-quality long reads can provide a complete picture of CYP2D6 for improved validation of complicated variants and haplotypes $[25,26]$. We demonstrate superior genotyping accuracy compared to other methods in 138 GeT-RM reference samples and 8 samples with PacBio HiFi data, covering 40 known star alleles. We applied this method to WGS data on 2504 unrelated samples from the 1000 Genomes Project [27] and report on the distribution of star alleles across five ethnic populations. This analysis expands the current understanding of the genetic diversity of CYP2D6, particularly on complex star alleles with SVs.

\section{Methods}

\section{Samples}

We included 138 GeT-RM reference samples in our truthset $[12,25]$. WGS was performed for 96 samples with TruSeq DNA PCR-free sample preparation and $2 \times 150 \mathrm{bp}$ reads sequenced on Illumina HiSeq $\mathrm{X}$ instruments. Genome build GRCh37 was used for read alignment with Isaac v04.16.09.24 [28]. The WGS data for the remaining 42 samples were downloaded as part of the 1000 Genomes Project (see below).

For population analysis, trio concordance tests and truthset comparison, we downloaded WGS BAM files from the 1000 Genomes Project (1kGP) (see "Data availability"). These BAM files were generated by sequencing $2 \times 150$ bp reads on Illumina NovaSeq 6000 instruments from PCR-free libraries sequenced to an averaged depth of at least $30 \times$ and aligned to the human reference, hs38DH, using BWA-MEM v0.7.15. The $1 \mathrm{kGP}$ data includes $347,661,504,503$, and 489 samples of Admixed American, African, East Asian, European and South Asian ancestry, respectively.

PacBio sequencing data for eight samples (Table S1) were downloaded from $1 \mathrm{kGP}$ and the Genome in a Bottle (GIAB) Consortium.

\section{CYP2D6 genotyping method used by Cyrius}

Read alignment accuracy is reduced in CYP2D6 because of the homology with CYP2D7 (Fig. 1) and this can make variant calling challenging and error prone. Cyrius uses a novel approach to overcome this challenge and a detailed workflow is described below and illustrated in Fig. 2 using NA12878 $(* 3 / * 68+* 4)$ as an example.

First, Cyrius identifies the total copies of both $C Y P 2 D 6$ and $C Y P 2 D 7$ combined (i.e., $\mathrm{CN}(C Y P 2 D 6+C Y P 2 D 7))$ following a similar method as previously described [29]. Read counts are calculated directly from the WGS aligned BAM file using all reads mapped to either CYP2D6 or CYP2D7, including reads aligned with a mapping quality of zero because of the homology between CYP2D6 and CYP2D7. The summed read count is normalized and

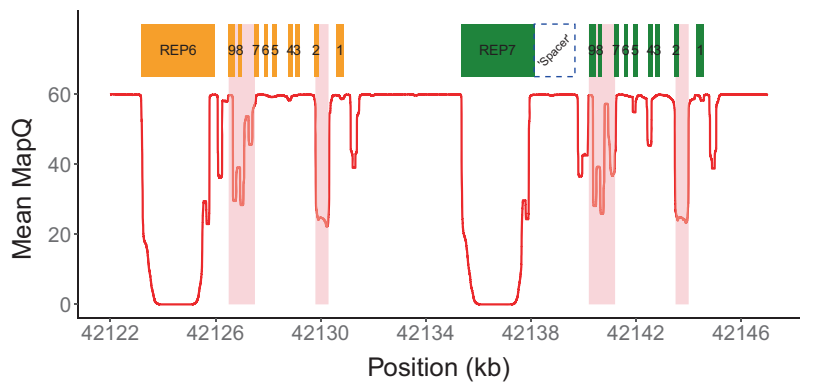

Fig. 1 WGS data quality in $C Y P 2 D 6 / C Y P 2 D 7$ region. Mean mapping quality (red line) averaged across $25041 \mathrm{kGP}$ samples plotted for each position in the $C Y P 2 D 6 / C Y P 2 D 7$ region (GRCh38). A median filter is applied in a $200 \mathrm{bp}$ window. The nine exons of CYP2D6/ $C Y P 2 D 7$ are shown as orange $(C Y P 2 D 6)$ and green $(C Y P 2 D 7)$ boxes. Two $2.8 \mathrm{~kb}$ repeat regions downstream of CYP2D6 (REP6, chr22:42123192-42125972) and CYP2D7 (REP7, chr22:4213534442138124) are near-identical and essentially unalignable. The purple dashed line box denotes the unique spacer region (chr22:4213812442139676) between CYP2D7 and REP7. Two major homology regions within the genes are shaded in pink and highlight areas of low mapping accuracy. 

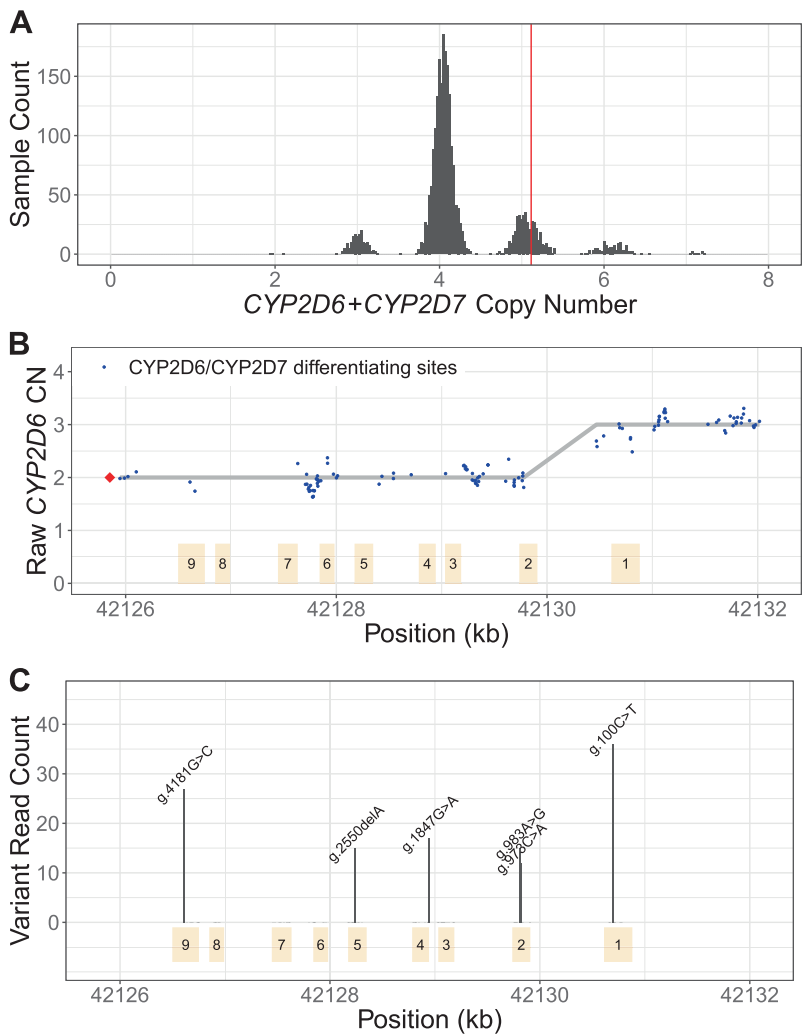

Fig. 2 Cyrius workflow, using NA12878 $(* 3 / * 68+* 4)$ as an example. A $\mathrm{CN}(C Y P 2 D 6+C Y P 2 D 7)$ is derived by counting and modeling all reads that align to either $C Y P 2 D 6$ or $C Y P 2 D 7$. The histogram shows the distribution of normalized $C Y P 2 D 6+C Y P 2 D 7$ depth in $25041 \mathrm{kGP}$ samples, showing peaks at $\mathrm{CN} 2,3,4,5,6$, and 7 . The red vertical line represents the value for NA12878, corresponding to CN5 that indicates an additional copy (could be CYP2D6 or hybrid). B SVs are called by examining the CNs of $C Y P 2 D 6 / C Y P 2 D 7$ differentiating bases. Exons are denoted by yellow boxes. Blue dots denote raw CYP2D6 $\mathrm{CNs}$, calculated as $\mathrm{CN}(C Y P 2 D 6+C Y P 2 D 7)$ multiplied by the ratio of CYP2D6 supporting reads out of CYP2D6 and CYP2D7 supporting reads. The red diamond denotes the $\mathrm{CN}$ of genes that are $C Y P 2 D 6-$ derived at the $3^{\prime}$ end (can be complete CYP2D6 or CYP2D7-CYP2D6 hybrid), calculated as $\mathrm{CN}(C Y P 2 D 6+C Y P 2 D 7)$ minus $\mathrm{CN}$ (spacer). The $C Y P 2 D 6 \mathrm{CN}$ is called at each $C Y P 2 D 6 / C Y P 2 D 7$ differentiating site and a change in $C Y P 2 D 6 \mathrm{CN}$ within the gene indicates the presence of a hybrid. In NA12878, the CYP2D6 CN changes from 2 to 3 between Exon 2 and Exon 1, indicating a CYP2D6-CYP2D7 hybrid $(* 68)$. C Supporting read counts of the star-allele defining protein-changing small variants are used to call the $\mathrm{CN}$ of each variant. The $y$ axis shows the read counts for all queried small variant positions. Six variants are called in NA12878, one of which, g.100C $>\mathrm{T}$, is called as two copies (one copy belongs to $* 4$ and the other belongs to $* 68$ ). Finally, star alleles are called based on detected SVs and small variants.

corrected for $\mathrm{GC}$ content and $\mathrm{CN}(C Y P 2 D 6+C Y P 2 D 7)$ is called from a Gaussian mixture model built on the normalized depth values. While there exist ambiguous alignments between CYP2D6 and CYP2D7, the sequencing coverage for both genes combined exhibits a clean signal (Fig. 2A), allowing us to identify SVs that result in a gain or loss in $\mathrm{CN}(C Y P 2 D 6+C Y P 2 D 7) \cdot \mathrm{CN}(C Y P 2 D 6+C Y P 2 D 7)$ is four in samples without $\mathrm{SV}$; a $\mathrm{CN}(C Y P 2 D 6+C Y P 2 D 7)$ of three suggests a deletion of either $C Y P 2 D 6$ or $C Y P 2 D 7$; a $\mathrm{CN}(C Y P 2 D 6+C Y P 2 D 7)$ of five suggests an extra copy, which could be a CYP2D6 duplication or a hybrid. The red vertical line in Fig. 2A shows the results for NA12878 where we identified five copies of $C Y P 2 D 6$ plus $C Y P 2 D 7$. We use the same approach to call the $\mathrm{CN}$ of the $1.6 \mathrm{~kb}$ spacer region between the repeat REP7 and CYP2D7 (Fig. 1). The $\mathrm{CN}$ (spacer) indicates the summed $\mathrm{CN}$ of $C Y P 2 D 7$ and $C Y P 2 D 6-C Y P 2 D 7$ hybrids. Thus, subtracting $\mathrm{CN}$ (spacer) from $\mathrm{CN}(C Y P 2 D 6+C Y P 2 D 7)$ gives the summed $\mathrm{CN}$ of $C Y P 2 D 6$ and $C Y P 2 D 7-C Y P 2 D 6$ hybrids.

We next determine the number of complete CYP2D6 genes as well as identify hybrid genes. To do this we identified 117 reliable bases that differ between CYP2D6 and CYP2D7 (Supplementary Information and Fig. S1) and use these to identify the exact form of SVs that impact CYP2D6. Cyrius estimates the CYP2D6 $\mathrm{CN}$ at each of the 117 $C Y P 2 D 6 / C Y P 2 D 7$ differentiating base positions. Based on $\mathrm{CN}(C Y P 2 D 6+C Y P 2 D 7)$, Cyrius calls the combination of $C Y P 2 D 6 \mathrm{CN}$ and $C Y P 2 D 7 \mathrm{CN}$ that produces the highest likelihood for the observed number of reads supporting CYP2D6- and CYP2D7-specific bases, as described previously [29]. Hybrids are identified when the $\mathrm{CN}$ of $C Y P 2 D 6$ changes within the gene. For example, NA12878 shown in Fig. 2B has two full copies of $C Y P 2 D 6$ and one hybrid where Exon 1 comes from CYP2D6 and Exons 2-9 come from CYP2D7 (i.e., *68).

Next Cyrius parses the read alignments to identify the protein-changing small variants that define star alleles and call their CNs (Fig. 2C). These variants are divided into two classes: (1) variants that fall in $C Y P 2 D 6 / C Y P 2 D 7$ homology regions, i.e., the shaded low mapping quality regions in Fig. 1, and (2) variants that occur in unique regions of CYP2D6. For the former, Cyrius looks for variant reads in CYP2D6 and its corresponding site in $C Y P 2 D 7$ to account for possible misalignments, e.g., a $C Y P 2 D 6$ read that aligns to $C Y P 2 D 7$. For the latter, Cyrius only uses the reads aligned to CYP2D6. The total CYP2D6 $\mathrm{CN}$ at the variant sites are taken into account during small variant calling so that a variant can be called at one copy, two copies or any $\mathrm{CN}$ less than or equal to the CYP2D6 $\mathrm{CN}$ at that site.

Finally, Cyrius matches the SVs and small variants against star-allele definitions (PharmVar, last accessed on 7/15/2020) and produces star-allele calls in diplotypes that are consistent with the called variants (Supplementary Information).

\section{Validating against truth from GeT-RM and long reads}

We confirmed that all the star alleles in our validation data are interrogated by Cyrius, Aldy, and Stargazer. When comparing the calls made by Cyrius, Aldy, and Stargazer 
against the truth genotypes, a genotype is considered a match as long as all star alleles in the truth genotype are present, even if the haplotype assignment is different. For example, several samples listed in GeT-RM as $* 1 / * 36+$ $* 36+* 10$ are called by Aldy as $* 1+* 36 / * 36+* 10$ and we considered these to be correct.

When validating genotype calls against the PacBio data, PacBio reads that cover the entire CYP2D6 gene (one single haplotype) were analyzed to identify small variants and the corresponding star allele. Reads carrying SVs were determined by aligning reads against a set of reference contigs that were constructed to represent known SVs $(* 5, * 13$, $* 36$, *68, and duplications).

\section{Running Aldy and Stargazer}

Aldy v2.2.5 was run using the command "aldy genotype $-\mathrm{p}$ illumina -g CYP2D6".

Stargazer v1.0.7 was run to genotype CYP2D6 using VDR as the control gene, with GDF and VCF files as input.
The 1kGP GeT-RM samples were originally aligned against GRCh38. Though Cyrius is designed to work on either GRCh38 or GRCh37, because Aldy and Stargazer currently only support GRCh37, for comparison between methods, these samples were realigned against GRCh37 using Isaac [28]. Note that Aldy v3.0, released (11/30/2020) after this paper was accepted, supports GRCh38 but we were not able to include it for testing in this paper.

\section{Results}

\section{Validation and performance comparison}

We compared the CYP2D6 calls made by Cyrius, Aldy, and Stargazer against 144 truthset samples, including 138 GeT-RM samples and eight samples with truth generated using PacBio HiFi sequence reads (two samples overlap between GeT-RM and PacBio, Table S1). Samples with SVs show distinct depth signals that allow us to call SVs accurately (Fig. 3). The long
Fig. 3 Depth patterns in samples with different types of SVs. Depth plots as described in Fig. 2B. CYP2D6 $\mathrm{CN}$ is called at each $C Y P 2 D 6 / C Y P 2 D 7$

differentiating site and a change in $C Y P 2 D 6 \mathrm{CN}$ within the gene indicates the presence of a hybrid. The depth profiles for different SV patterns are shown in NA19239 (no SV), HG02465 (deletion, *5), HG01624 (duplication), HG01161 (CYP2D7-CYP2D6 hybrid, *13), NA24631 (CYP2D6CYP2D7 hybrid, *36), NA12878 (CYP2D6-CYP2D7 hybrid, *68), HG00290 (tandem arrangement $* 13+* 2$ ), and NA19982 (two different SVs, $* 13$ and $* 68$, one on each haplotype). The hybrids in NA24631 and NA12878 are confirmed with PacBio reads in Fig. 4.

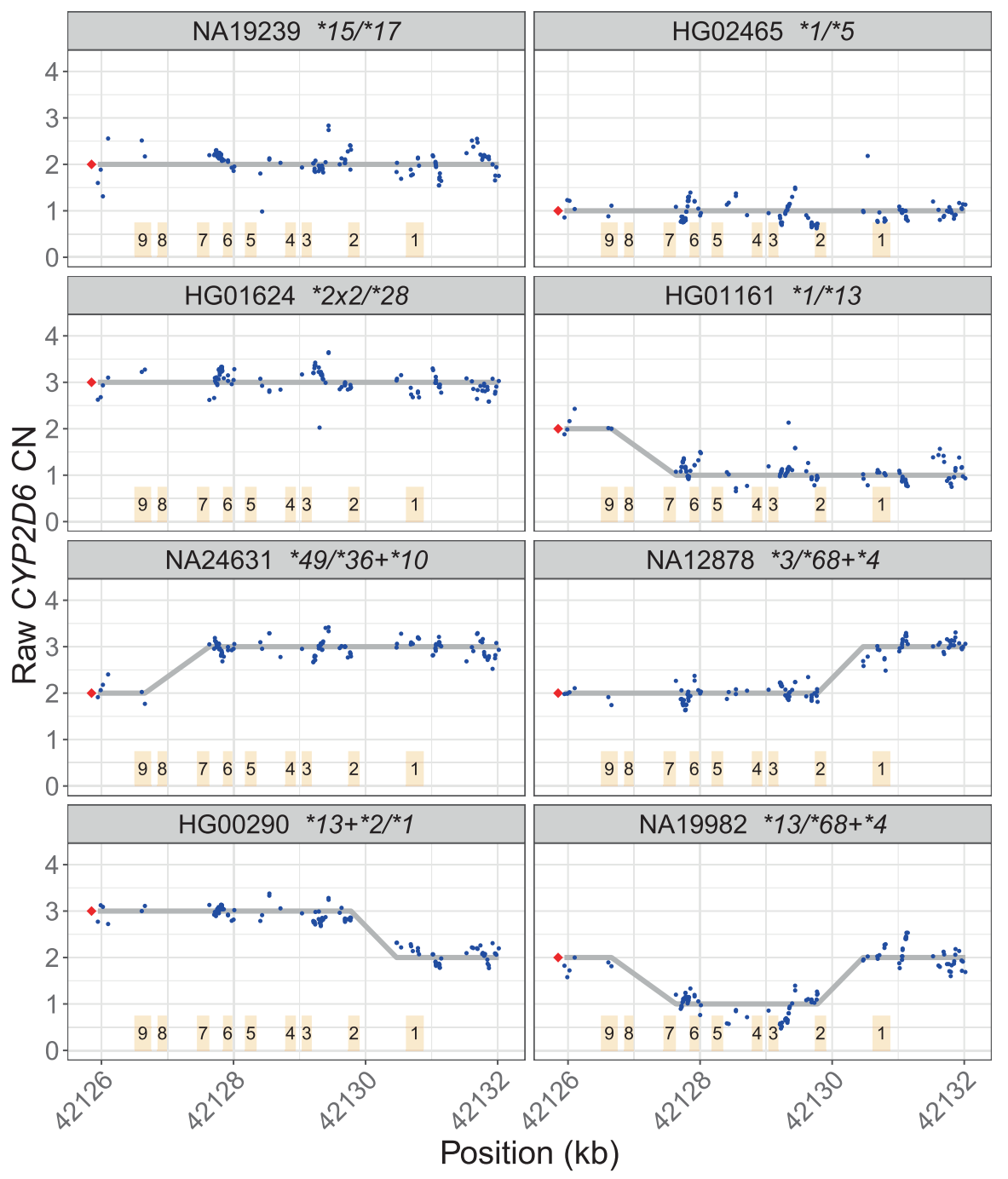


Reference Genome
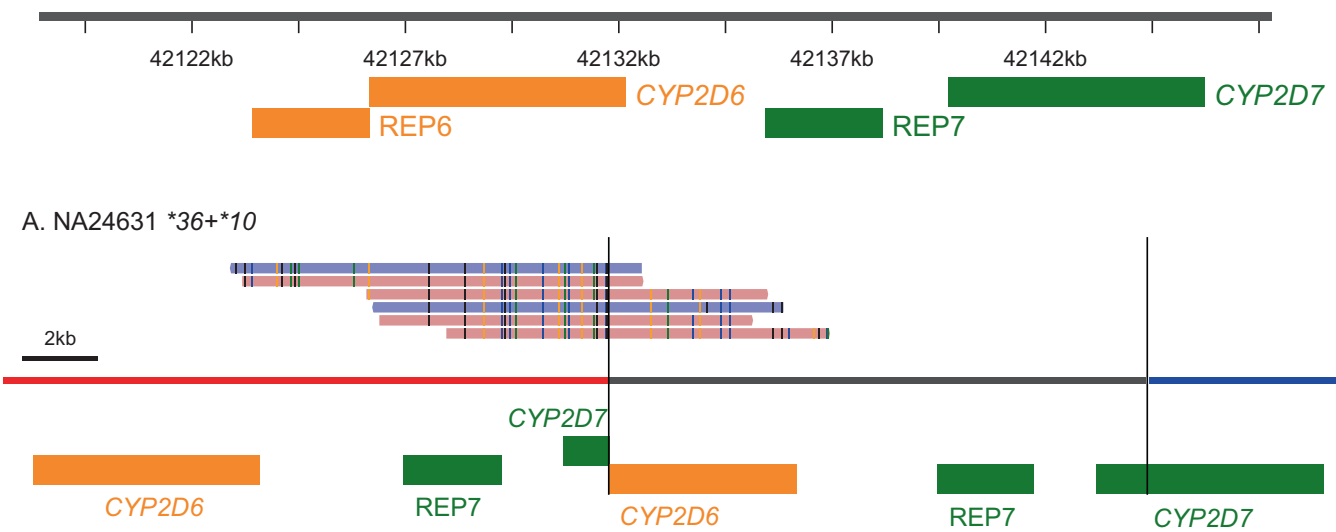

B. NA12878*68+*4

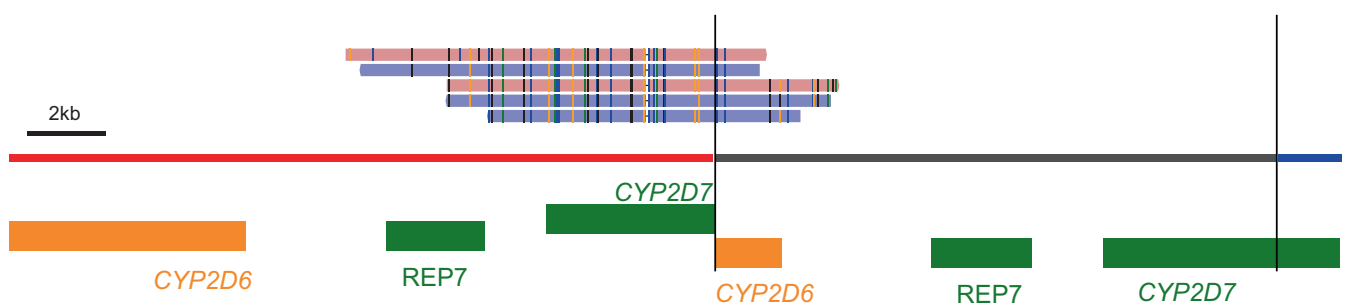

Fig. 4 Structural variants validated by PacBio HiFi reads. PacBio reads supporting CYP2D6-CYP2D7 hybrid $* 36$ and $* 68$, confirming SVs called in NA24631 and NA12878 (third row, Fig. 3). PacBio reads were realigned against modified sequence contigs representing

the hybrids and plotted using sv-viz2 [42]. The black vertical lines mark the boundaries of the duplicated sequences, represented by the gray region. The red and blue regions represent flanking sequences.

Table 1 Summary of benchmarking results against truth in 144 samples.

\begin{tabular}{lllllllll}
\hline Caller & $\begin{array}{l}\text { Total } \\
\text { concordant }\end{array}$ & Concordance & $\begin{array}{c}\text { Deletion } \\
N=15\end{array}$ & $\begin{array}{c}\text { Duplication } \\
N=13\end{array}$ & $\begin{array}{c}\text { Hybrid } \\
N=26\end{array}$ & $\begin{array}{l}\text { No SV } \\
N=90\end{array}$ & $\begin{array}{l}\text { Concordance, samples } \\
\text { with SV }\end{array}$ & $\begin{array}{l}\text { Concordance, samples } \\
\text { without SV }\end{array}$ \\
\hline Cyrius & $139^{\mathrm{a}}$ & $96.5 \%$ & 14 & 12 & 25 & 88 & $94.4 \%$ & $97.8 \%$ \\
Aldy & 125 & $86.8 \%$ & 13 & 11 & 23 & 78 & $87.0 \%$ & $86.7 \%$ \\
Stargazer & 121 & $84.0 \%$ & 14 & 10 & 17 & 80 & $75.9 \%$ & $88.9 \%$ \\
\hline
\end{tabular}

${ }^{\mathrm{a}}$ Cyrius has since been improved and can correctly call the CYP2D6 diplotype of 143 (99.3\%) of these 144 samples.

reads allow us to locate and visualize breakpoints of the common SVs in the region (Fig. 4) and thus serve as a valuable resource for studying complex star alleles and confirm the phasing of the variants for the star alleles.

Comparing against the GeT-RM samples, we found three samples where the calls of all three software methods agree with each other but disagree with the GeT-RM consensus (Table S1). First, for NA18519, the WGS-based genotype is $* 106 / * 29$ with reads carrying the variant defining *106 (Fig. S2). This genotype is also confirmed by other studies $[23,24]$. The GeT-RM consensus is $* 1 / * 29$, because none of the GeT-RM assays interrogated $* 106$ and the sample was not sequenced. The remaining two samples, NA23874 and NA24008, have the *68 CYP2D6-CYP2D7 hybrid that is not represented in the GeT-RM consensus. For these, the depth profiles show a $\mathrm{CN}$ gain in Exon 1 (Fig. S3A) and
PacBio long reads confirm the presence of $* 68$ hybrid (Fig. S3B/C). In GeT-RM testing, these two samples only underwent limited CNV testing (no TaqMan CNV result is available for Exon 1, the CYP2D6 part of the hybrid). Therefore, based on this additional evidence, the GeT-RM truth genotypes for these two samples should be updated to include $* 68$. For the accuracy calculations below, we consider these three samples to be correctly genotyped by the WGS-based methods.

Cyrius initially made five discordant calls in the 144 truth samples, showing a concordance of $96.5 \%$ (Table 1). We were subsequently able to identify the causes and improve Cyrius to correctly call 4 of these 5 samples (Supplementary Information, Figs. S4-S6, Table S2), reaching a "trained" concordance of $99.3 \%$ (143 out of 144 samples). In contrast, both of the other $C Y P 2 D 6$ callers had concordance 
less than $90 \%$. Aldy had a concordance of $86.8 \%$ and, in particular, overcalled several hybrids such as $* 61, * 63, * 78$, and $* 83$ (called in 7 out of 19 discordant samples, Table S1), even in samples without SVs. Stargazer had a concordance of $84 \%$ and is most prone to errors when SVs are present. The concordance in samples with SVs is $75.9 \%$, and 13 out of the 23 discordant calls are in samples with SVs (Table 1). Using the CPIC-recommended method for translation of CYP2D6 genotype to phenotype [7], the concordances between the truth phenotypes and those predicted for Cyrius, Aldy, and Stargazer are 97.9\% (99.3\% after improvement), $89.6 \%$, and $90.3 \%$, respectively (Table S1). An analysis of genotyping accuracy at lower sequencing depths $(<30 \times)$ is included in Supplementary Information (Fig. S7, Table S3).

Together, the validation samples used in this study confirmed our CYP2D6 calling accuracy in 47 distinct haplotypes (Table 2), including 40 star alleles as well as several SV structures, such as duplications and tandem arrangements including $* 13+* 2, * 68+* 4, * 36+* 10$, and $* 36+* 36+$ $* 10$. Of these, *49 is not found in GeT-RM but present in a sample with PacBio data. These 40 star alleles represent $30.5 \%$ of the 131 star alleles in PharmVar and 51.7\% (31 out of 60) of the star alleles with known function.

We next assessed Mendelian consistency of the Cyrius calls in sequencing data from 597 trios (Table S4 and Supplementary Information). While the comparison above against truth genotypes allows for different haplotype phasing, the Mendelian consistency check is a more stringent check of the phasing of the star alleles when more than two copies of CYP2D6 are present. Of the 572 trios with calls in all three family members, $561(98.1 \%)$ are Mendelian consistent. All of the inconsistent trios could be resolved by changing the phasing-i.e., no proband had a called star allele that was absent in both parents. The majority (8/11) of the inconsistent cases are where the trio identified that two identical copies of CYP2D6 should be on the same haplotype with the other haplotype having zero copy of CYP2D6 (i.e., *Cyrius call $* 1 / * 1$ vs. trio-based phasing $* 5 / * 1 \times 2$ ). This Mendelian consistency check confirms the consistency of the genotypes across the pedigree but not the accuracy of the star alleles called. Combining the trio concordance tests with the accuracy tests performed above against truth genotypes provides confidence in the overall accuracy of the genotypes produced by Cyrius.

\section{CYP2D6 haplotype frequencies across five ethnic populations}

We next looked beyond the validation samples to study CYP2D6 in the global population. For this, we analyzed the haplotype distribution by population (Europeans, Africans,
East Asians, South Asians, and admixed Americans) in 2504 unrelated 1kGP samples (Fig. 5, Tables 2, S5). Additionally, the predicted phenotype frequencies for these populations are illustrated in Fig. S8. Cyrius made definitive diplotype calls in 2456 (98.1\%) of the samples calling 52 distinct star alleles (The 48 no-calls are explained in Supplementary Information). Of these 52 star alleles, 40 overlapped star alleles that had been included in our validation data. These 40 alleles represent $96 \%$ of all the star alleles called in the $1 \mathrm{kGP}$ samples (Table 2).

The haplotype frequencies mostly agree (correlation coefficient $0.79-0.97$ ) with the summary of published allele frequencies in PharmGKB $[6,30]$ (Fig. 5B, PharmGKB last accessed on $5 / 1 / 2020$ ). While we report similar frequencies for CYP2D6 deletion or duplication alleles as in PharmGKB, we report a higher frequency than PharmGKB for the SVcontaining haplotype $* 36+* 10$ in East Asians and another $\mathrm{SV} * 68+* 4$ in Europeans (Fig. 5B, dots annotated in red). Previously reported frequencies of $* 36+* 10$ in East Asians fall into a wide range (10-35\%) [31-36], reflecting the variability in $\mathrm{CNV}$ testing across assays. Additionally, $* 68$ is often not interrogated in many studies, and it has been suggested that $>20 \%$ of reported $* 4$ alleles are actually in tandem with $* 68[37,38]$. Together, we estimate that the frequencies of haplotypes involving SVs are 38.6\%, 11.2\%, 11.4\%, 6.8\%, and $7 \%$ in East Asians, Europeans, Africans, Americans, and South Asians, respectively, and are 5.9\%, 5.9\%, 1.9\%, 1.6\%, and $0.9 \%$ higher than reported in the literature and summarized by PharmGKB.

There are a few other star alleles for which we report a lower frequency than PharmGKB (Fig. 5B, dots annotated in blue), highlighting the difficulty of merging data from multiple studies using different technologies [4]. These include *2 in all five populations. Since $* 2$ is the default allele assignment for variant $2851 \mathrm{C}>\mathrm{T}$ and $4181 \mathrm{G}>\mathrm{C}$ unless additional variants defining other star alleles are interrogated, its frequency is likely overestimated in the literature [6]. Similarly, *10 is likely overestimated [4] in East Asians and South Asians and *4 is likely overestimated [37] in Europeans, particularly because a fraction of reported $* 10$ or $* 4$ alleles are $* 36+* 10$ or $* 68+* 4$. It should be noted that since the CYP2D6 enzyme activity is identical between $* 10$ and $* 36+$ $* 10$ and between $* 4$ and $* 68+* 4$, this overestimation has no clinical impact. Finally, we report a lower frequency for $* 41$ in Africans. Since this allele has not been identified consistently by its defining SNP across studies, it is likely overestimated in Africans [30, 39, 40].

\section{Discussion}

We present a new software tool, Cyrius, that accurately genotypes the highly complex CYP2D6 region. Using 
Table 2 Haplotypes validated in this study and their frequencies in 1kGP.

\begin{tabular}{|c|c|c|c|c|c|c|c|c|c|}
\hline Haplotype & $\begin{array}{l}\text { Pan- } \\
\text { ethnic } \\
(N= \\
2504)\end{array}$ & $\begin{array}{l}\text { European } \\
(N=503)\end{array}$ & $\begin{array}{l}\text { Admixed } \\
\text { American } \\
(N=347)\end{array}$ & $\begin{array}{l}\text { East Asian } \\
(N=504)\end{array}$ & $\begin{array}{l}\text { African } \\
(N=661)\end{array}$ & $\begin{array}{l}\text { South Asian } \\
(N=489)\end{array}$ & $\begin{array}{l}\text { Validated in } \\
\text { this study }\end{array}$ & $\begin{array}{l}\text { In GeT- } \\
\text { RM } \\
\text { full set }\end{array}$ & $\begin{array}{l}\text { CPIC clinical } \\
\text { allele function }\end{array}$ \\
\hline$* 1$ & 33.35 & 35.88 & 45.97 & 26.09 & 25.87 & 39.37 & $\mathrm{x}$ & $\mathrm{x}$ & Normal \\
\hline$* 2$ & 14.76 & 15.9 & 18.59 & 7.74 & 12.71 & 20.86 & $\mathrm{x}$ & $\mathrm{x}$ & Normal \\
\hline$* 3$ & 0.54 & 1.79 & 0.58 & 0 & 0.23 & 0.2 & $\mathrm{x}$ & $\mathrm{x}$ & No \\
\hline$* 4$ & 5.93 & 11.83 & 9.22 & 0.2 & 2.34 & 8.28 & $\mathrm{x}$ & $\mathrm{x}$ & No \\
\hline$* 5$ & 3.49 & 2.39 & 2.02 & 3.47 & 5.82 & 2.56 & $\mathrm{x}$ & $\mathrm{x}$ & No \\
\hline$* 6$ & 0.5 & 2.09 & 0.29 & 0 & 0.08 & 0.1 & $\mathrm{x}$ & $\mathrm{x}$ & No \\
\hline$* 7$ & 0.18 & 0 & 0 & 0 & 0 & 0.92 & $\mathrm{x}$ & $\mathrm{x}$ & No \\
\hline$* 9$ & 0.68 & 2.39 & 1.3 & 0 & 0.08 & 0 & $\mathrm{x}$ & $\mathrm{x}$ & Decreased \\
\hline$* 10$ & 5.25 & 1.39 & 1.44 & 14.98 & 3.86 & 3.78 & $\mathrm{x}$ & $\mathrm{x}$ & Decreased \\
\hline$* 11$ & 0.02 & 0 & 0 & 0 & 0.08 & 0 & $\mathrm{x}$ & $\mathrm{x}$ & No \\
\hline$* 13$ & 0.1 & 0.2 & 0.14 & 0 & 0.08 & 0.1 & $\mathrm{x}$ & $\mathrm{x}$ & No \\
\hline$* 14$ & 0.18 & 0 & 0 & 0.89 & 0 & 0 & $\mathrm{x}$ & $\mathrm{x}$ & Decreased \\
\hline$* 15$ & 0.06 & 0 & 0 & 0 & 0.23 & 0 & $\mathrm{x}$ & $\mathrm{x}$ & No \\
\hline$* 17$ & 5.25 & 0.2 & 0.86 & 0 & 19.29 & 0 & $\mathrm{x}$ & $\mathrm{x}$ & Decreased \\
\hline$* 21$ & 0.1 & 0 & 0 & 0.5 & 0 & 0 & $\mathrm{x}$ & $\mathrm{x}$ & No \\
\hline$* 22$ & 0.06 & 0.3 & 0 & 0 & 0 & 0 & $\mathrm{x}$ & $\mathrm{x}$ & Uncertain \\
\hline$* 27$ & 0.12 & 0 & 0.14 & 0 & 0.38 & 0 & & & Normal \\
\hline$* 28$ & 0.12 & 0.5 & 0.14 & 0 & 0 & 0 & $\mathrm{x}$ & $\mathrm{x}$ & Uncertain \\
\hline$* 29$ & 2.64 & 0 & 0.29 & 0 & 9.83 & 0 & $\mathrm{x}$ & $\mathrm{x}$ & Decreased \\
\hline$* 31$ & 0.12 & 0.2 & 0.58 & 0 & 0 & 0 & $\mathrm{x}$ & $\mathrm{x}$ & No \\
\hline$* 32$ & 0.08 & 0.3 & 0.14 & 0 & 0 & 0 & & & Uncertain \\
\hline$* 33$ & 0.18 & 0.6 & 0.29 & 0 & 0 & 0.1 & $\mathrm{x}$ & $\mathrm{x}$ & Normal \\
\hline$* 34$ & 0.02 & 0 & 0 & 0 & 0.08 & 0 & & & Normal \\
\hline$* 35$ & 1.48 & 4.67 & 2.59 & 0 & 0.23 & 0.61 & $\mathrm{x}$ & $\mathrm{x}$ & Normal \\
\hline$* 36$ & 0.26 & 0 & 0 & 0.2 & 0.83 & 0 & & & No \\
\hline *39 & 0.08 & 0 & 0.14 & 0 & 0.08 & 0.2 & & $\mathrm{x}$ & Normal \\
\hline$* 40$ & 0.24 & 0 & 0 & 0 & 0.91 & 0 & $\mathrm{x}$ & $\mathrm{x}$ & No \\
\hline$* 41$ & 6.07 & 8.75 & 5.91 & 3.77 & 1.59 & 11.86 & $\mathrm{x}$ & $\mathrm{x}$ & Decreased \\
\hline$* 43$ & 0.5 & 0.1 & 0 & 0 & 1.06 & 1.02 & $\mathrm{x}$ & $\mathrm{x}$ & Uncertain \\
\hline$* 45$ & 0.88 & 0 & 0.29 & 0 & 3.18 & 0 & $\mathrm{x}$ & $\mathrm{x}$ & Normal \\
\hline$* 46$ & 0.16 & 0 & 0.14 & 0 & 0.53 & 0 & $\mathrm{x}$ & $\mathrm{x}$ & Normal \\
\hline$* 49$ & 0.1 & 0 & 0 & 0.5 & 0 & 0 & $\mathrm{x}$ & & Decreased \\
\hline$* 52$ & 0.02 & 0 & 0 & 0.1 & 0 & 0 & $\mathrm{x}$ & $\mathrm{x}$ & Uncertain \\
\hline$* 56$ & 0.02 & 0 & 0 & 0 & 0.08 & 0 & $\mathrm{x}$ & $\mathrm{x}$ & No \\
\hline$* 59$ & 0.06 & 0.2 & 0.14 & 0 & 0 & 0 & $\mathrm{x}$ & $\mathrm{x}$ & Decreased \\
\hline$* 68$ & 0.04 & 0 & 0 & 0 & 0.08 & 0.1 & & & No \\
\hline$* 71$ & 0.12 & 0 & 0 & 0.6 & 0 & 0 & $\mathrm{x}$ & $\mathrm{x}$ & Uncertain \\
\hline$* 82$ & 0.06 & 0 & 0.43 & 0 & 0 & 0 & $\mathrm{x}$ & $\mathrm{x}$ & Unknown \\
\hline$* 83$ & 0.02 & 0 & 0 & 0 & 0 & 0.1 & & $\mathrm{x}$ & Uncertain \\
\hline$* 84$ & 0.02 & 0 & 0 & 0 & 0.08 & 0 & & & Uncertain \\
\hline$* 86$ & 0.44 & 0 & 0 & 0 & 0 & 2.25 & & & Unknown \\
\hline *99 & 0.04 & 0 & 0 & 0 & 0 & 0.2 & $\mathrm{x}$ & $\mathrm{x}$ & No \\
\hline *106 & 0.32 & 0 & 0.14 & 0 & 1.13 & 0 & $\mathrm{x}$ & $\mathrm{x}$ & Uncertain \\
\hline *108 & 0.06 & 0.3 & 0 & 0 & 0 & 0 & & $\mathrm{x}$ & Unknown \\
\hline$* 111$ & 0.16 & 0 & 0 & 0 & 0 & 0.82 & $\mathrm{x}$ & $\mathrm{x}$ & Unknown \\
\hline
\end{tabular}


Table 2 (continued)

\begin{tabular}{|c|c|c|c|c|c|c|c|c|c|}
\hline Haplotype & $\begin{array}{l}\text { Pan- } \\
\text { ethnic } \\
(N= \\
2504)\end{array}$ & $\begin{array}{l}\text { European } \\
(N=503)\end{array}$ & $\begin{array}{l}\text { Admixed } \\
\text { American } \\
(N=347)\end{array}$ & $\begin{array}{l}\text { East Asian } \\
(N=504)\end{array}$ & $\begin{array}{l}\text { African } \\
(N=661)\end{array}$ & $\begin{array}{l}\text { South Asian } \\
(N=489)\end{array}$ & $\begin{array}{l}\text { Validated in } \\
\text { this study }\end{array}$ & $\begin{array}{l}\text { In GeT- } \\
\text { RM } \\
\text { full set }\end{array}$ & $\begin{array}{l}\text { CPIC clinical } \\
\text { allele function }\end{array}$ \\
\hline$* 112$ & 0.04 & 0 & 0 & 0 & 0 & 0.2 & $\mathrm{x}$ & $\mathrm{x}$ & Unknown \\
\hline$* 113$ & 0.16 & 0 & 0 & 0 & 0 & 0.82 & $\mathrm{x}$ & $\mathrm{x}$ & Unknown \\
\hline$* 117$ & 0.08 & 0.4 & 0 & 0 & 0 & 0 & & & Unknown \\
\hline$* 121$ & 0.02 & 0 & 0 & 0 & 0.08 & 0 & & & Unknown \\
\hline$* 125$ & 0.1 & 0 & 0 & 0 & 0.38 & 0 & & & Unknown \\
\hline$* 139$ & 0.02 & 0 & 0 & 0 & 0.08 & 0 & & & Unknown \\
\hline$* 1 \times 2$ & 0.54 & 0.5 & 1.44 & 0.1 & 0.45 & 0.51 & $\mathrm{x}$ & $\mathrm{x}$ & Increased \\
\hline$* 1 \times 3$ & 0.02 & 0 & 0 & 0 & 0.08 & 0 & & & Increased \\
\hline$* 2 \times 2$ & 1.14 & 1.49 & 0.58 & 0.6 & 2.12 & 0.41 & $\mathrm{x}$ & $\mathrm{x}$ & Increased \\
\hline$* 2 \times 3$ & 0.04 & 0.1 & 0 & 0 & 0.08 & 0 & & & Increased \\
\hline$* 4 \times 2$ & 0.88 & 0.3 & 0.14 & 0 & 3.03 & 0 & $\mathrm{x}$ & $\mathrm{x}$ & No \\
\hline$* 4 \times 3$ & 0.04 & 0 & 0 & 0 & 0.15 & 0 & & & No \\
\hline$* 9 \times 2$ & 0.02 & 0.1 & 0 & 0 & 0 & 0 & & & Normal \\
\hline$* 10 \times 2$ & 0.06 & 0 & 0 & 0.3 & 0 & 0 & $\mathrm{x}$ & $\mathrm{x}$ & Decreased \\
\hline$* 17 \times 2$ & 0.02 & 0 & 0 & 0 & 0.08 & 0 & & $\mathrm{x}$ & Normal \\
\hline$* 29 \times 2$ & 0.1 & 0 & 0 & 0 & 0.38 & 0 & & & Normal \\
\hline$* 35 \times 2$ & 0.02 & 0 & 0.14 & 0 & 0 & 0 & & & Increased \\
\hline$* 43 \times 2$ & 0.04 & 0 & 0.14 & 0 & 0.08 & 0 & & & Unknown \\
\hline$* 45 \times 3$ & 0.02 & 0 & 0 & 0 & 0.08 & 0 & & & Increased \\
\hline$* 36+* 10$ & 7.15 & 0 & 0.14 & 34.13 & 0.08 & 1.23 & $\mathrm{x}$ & $\mathrm{x}$ & Decreased \\
\hline$* 36+* 36$ & 0.04 & 0 & 0 & 0.2 & 0 & 0 & & & No \\
\hline$* 68+* 4$ & 1.94 & 5.57 & 2.45 & 0 & 0.23 & 2.15 & $\mathrm{x}$ & $\mathrm{x}$ & No \\
\hline$* 68+* 68+* 4$ & 0.08 & 0.1 & 0.43 & 0 & 0 & 0 & & & No \\
\hline$* 36+* 36+* 10$ & 0.36 & 0 & 0 & 1.79 & 0 & 0 & $\mathrm{x}$ & $\mathrm{x}$ & Decreased \\
\hline $\begin{array}{l}* 36+* 36+* 36+ \\
* 10\end{array}$ & 0.02 & 0 & 0 & 0.1 & 0 & 0 & $\mathrm{x}$ & $\mathrm{x}$ & Decreased \\
\hline$* 13+* 2$ & 0.1 & 0.2 & 0.43 & 0 & 0 & 0 & $\mathrm{x}$ & $\mathrm{x}$ & Normal \\
\hline$* 4.013+* 4$ & 0.14 & 0.7 & 0 & 0 & 0 & 0 & & $\mathrm{x}$ & No \\
\hline$* 1+* 90$ & 0.02 & 0 & 0 & 0.1 & 0 & 0 & $\mathrm{x}$ & $\mathrm{x}$ & Uncertain \\
\hline $\begin{array}{l}* 36+* 36+* 83+ \\
* 10\end{array}$ & 0.02 & 0 & 0 & 0.1 & 0 & 0 & & & Uncertain \\
\hline Unknown & 1.92 & 0.6 & 2.31 & 3.57 & 1.97 & 1.23 & & & \\
\hline $\begin{array}{l}\text { \% Haplotypes } \\
\text { overlapping the } \\
\text { validation set }\end{array}$ & 96.1 & 97.4 & 96.5 & 95.9 & 95.1 & 96.1 & & & \\
\hline
\end{tabular}

144 samples, including 8 with long read data, as an orthogonal validation dataset, we show that Cyrius outperforms other CYP2D6 callers, achieving $96.5 \%$ concordance versus $86.8 \%$ for Aldy and $84 \%$ for Stargazer. In particular, by using a novel $\mathrm{CN}$ calling approach, selecting a set of reliable $C Y P 2 D 6 / C Y P 2 D 7$ differentiating sites and accounting for possible misaligned reads, Cyrius is able to accurately identify star alleles with SVs, achieving $94.4 \%$ concordance compared to $87 \%$ for Aldy and $75.9 \%$ for Stargazer. Our comparison against the truth set allows us to identify ways to improve the accuracy of Cyrius and after implementing those changes, we are able to increase the overall concordance to $99.3 \%$ (from $96.5 \%$ ) and to $100 \%$ (from $94.4 \%$ ) for the samples with SVs. We estimate that the star alleles miscalled in the validation data $(* 40, * 46, * 56$ and $* 36$ singleton) are only present in $\sim 0.68 \%$ of the population. Therefore, Cyrius's accuracy is likely even higher in the population.

Across the 144 validation samples, we are able to confirm the accuracy of Cyrius across 40 different star alleles 
Fig. 5 CYP2D6 allele frequencies across five ethnic populations. A Ten most common haplotypes with altered CYP2D6 function. Those with increased function are labeled in red, those with no function in black and those with decreased function in blue. B Comparison between 1kGP and PharmGKB frequencies. Each dot represents a haplotype with a frequency $\geq$ $0.5 \%$ in either $1 \mathrm{kGP}$ or PharmGKB. SV-related haplotypes are marked in red, including the two haplotypes with the largest deviation $(* 36+* 10$ in East Asians and $* 68+* 4$ in Europeans). Other haplotypes with deviated values are annotated in blue. A diagonal line is drawn for each panel. Correlation coefficients are listed for each population.

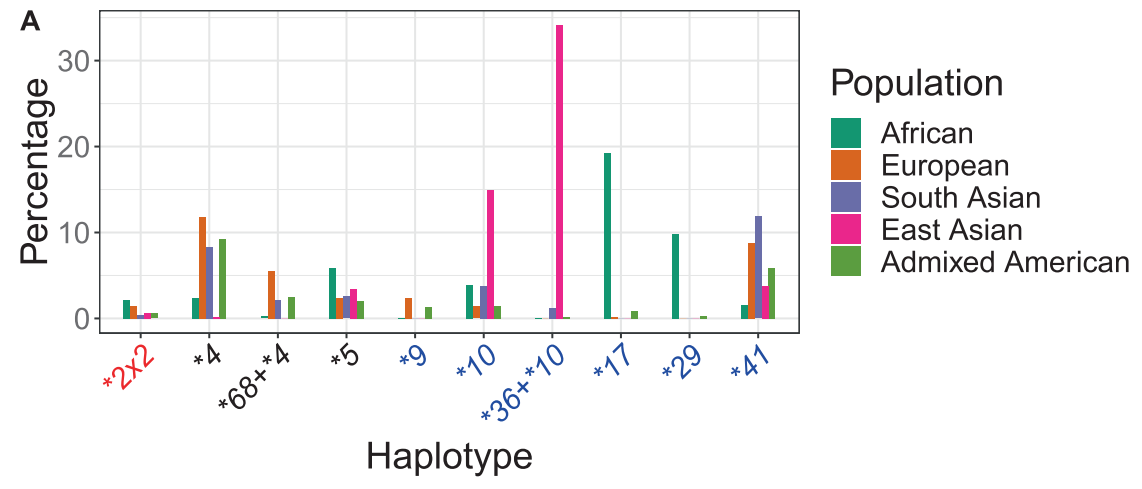

B

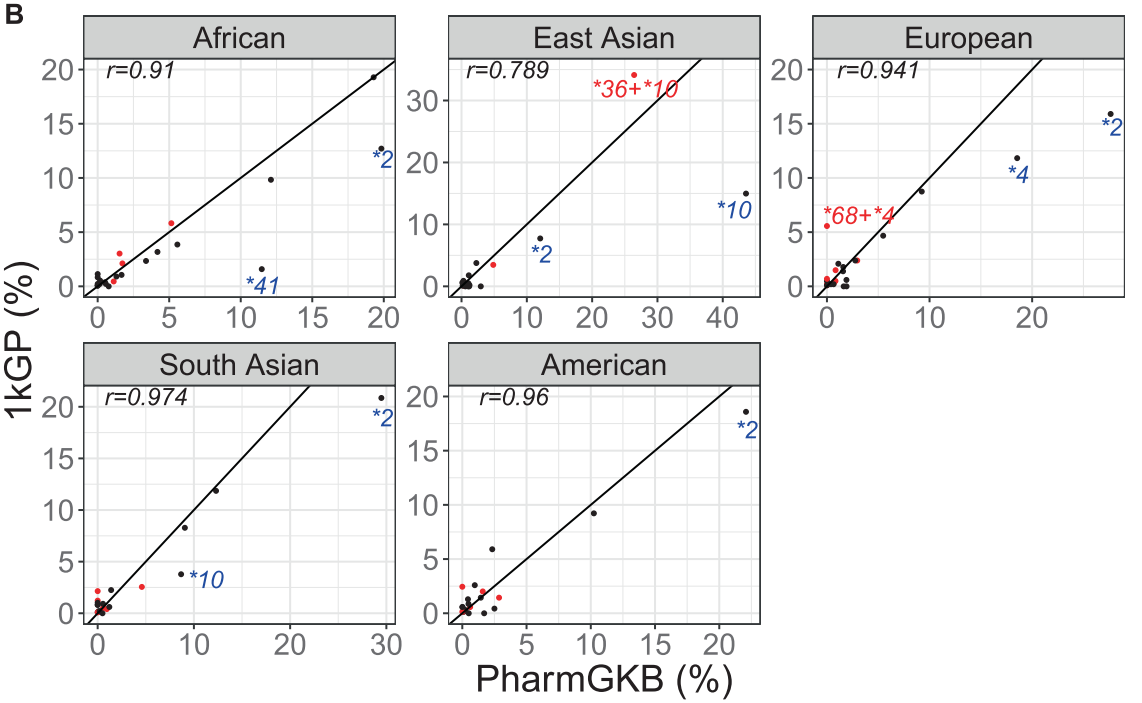

that represent roughly $96 \%$ of the star alleles in the pangenomic $1 \mathrm{kGP}$ population. In general, the allele frequencies we calculate for the five ethnic populations agree with previous studies for single copy star alleles. There are a number of limitations in the accuracy of the allele frequencies in PharmGKB because most studies test for a limited set of variants and there is often inadequate testing of CNVs [4, 6]. WGS provides a promising option for building up more accurate population frequency databases because it assays all of the variants including CNVs and, combined with the right software, is able to resolve almost all of the known star alleles accurately. Furthermore, when new star alleles are added, it is easy to update allele frequencies by reanalyzing the same WGS data without retesting that may require a new assay design.

In our analysis of the $1 \mathrm{kGP}$ samples, Cyrius is able to call a definitive genotype in $98.1 \%$ of the samples. A future direction is to better understand the $1.9 \%$ of the samples that were not called and improve our algorithm so that it can also resolve these genotypes. For example, in samples where multiple haplotype configurations are possible, it could be useful to take a probabilistic approach to derive the most likely genotype given the observed variants. In addition, one limitation of this study is that there is no truth data available to validate the remaining, rarer star alleles defined by PharmVar. Continuing to sequence and test more samples will help confirm our ability to genotype rare star alleles and will also identify additional variants that can be used to distinguish ambiguous diplotypes.

WGS provides a unique opportunity to profile all genetic variations for the entire genome but many clinically important regions/variants are beyond the ability of most secondary analysis pipelines. CYP2D6 is among the difficult regions in the genome that are both clinically important and also require specialized informatics solutions to supplement generic WGS pipelines. Such targeted methods have already been applied successfully to some difficult regions, such as repeat expansions [41] and the SMN1 gene [29] responsible for spinal muscular atrophy. The method employed in Cyrius can be applied to resolve other paralogs that suffer from the same homology problem. We are currently extending this method to genotype other pharmacogenes with a paralog, $C Y P 2 A 6$, and $C Y P 2 B 6$, and will apply this method to more genes in the future. With the continued development of more targeted methods like Cyrius, we can help accelerate pharmacogenomics and move one step closer towards personalized medicine. 


\section{Data availability}

Cyrius can be downloaded from: https://github.com/ Illumina/Cyrius. The $1 \mathrm{kGP}$ data can be downloaded from https://www.ncbi.nlm.nih.gov/bioproject/PRJEB31736/ and https://www.ncbi.nlm.nih.gov/bioproject/PRJEB36890/.

WGS data for 70 GeT-RM samples can be downloaded from: https://www.ebi.ac.uk/ena/data/view/PRJEB19931. For NA12878, NA24385, and NA24631, the PacBio Sequel II data is available in SRA under PRJNA540705, PRJNA529679, and PRJNA540706, and the Illumina data is available in ENA under PRJEB35491. For the remaining five samples with PacBio truth, the PacBio Sequel II data is available from http://ftp.1000genomes.ebi.ac.uk/voll/ftp/da ta_collections/HGSVC2/working/.

Acknowledgements We thank the CDC Genetic Testing Reference Material Program (GeT-RM) for generating the consensus genotypes. We thank the New York Genome Center and the Coriell Institute for Medical Research for generating and releasing the 1kGP WGS data.

\section{Compliance with ethical standards}

Conflict of interest XC, FS, NG, AM, CR, RJT, DRB, and MAE are employees of Illumina Inc.

Publisher's note Springer Nature remains neutral with regard to jurisdictional claims in published maps and institutional affiliations.

Open Access This article is licensed under a Creative Commons Attribution 4.0 International License, which permits use, sharing, adaptation, distribution and reproduction in any medium or format, as long as you give appropriate credit to the original author(s) and the source, provide a link to the Creative Commons license, and indicate if changes were made. The images or other third party material in this article are included in the article's Creative Commons license, unless indicated otherwise in a credit line to the material. If material is not included in the article's Creative Commons license and your intended use is not permitted by statutory regulation or exceeds the permitted use, you will need to obtain permission directly from the copyright holder. To view a copy of this license, visit http://creativecommons. org/licenses/by/4.0/.

\section{References}

1. Evans WE, Relling MV. Moving towards individualized medicine with pharmacogenomics. Nature. 2004;429:464-8. https://doi.org/ 10.1038/nature02626.

2. Zhou S-F. Polymorphism of human cytochrome P450 2D6 and its clinical significance: Part I. Clin Pharmacokinet. 2009;48:689-723. https://doi.org/10.2165/11318030-000000000-00000.

3. Gaedigk A, Ingelman-Sundberg M, Miller NA, Leeder JS, Whirl-Carrillo M, Klein TE, et al. The Pharmacogene Variation (PharmVar) Consortium: incorporation of the human cytochrome P450 (CYP) allele nomenclature database. Clin Pharmacol Ther. 2018;103:399-401. https://doi.org/10.1002/cpt.910.

4. Nofziger C, Turner AJ, Sangkuhl K, Whirl-Carrillo M, Agúndez JAG, Black JL, et al. PharmVar GeneFocus: CYP2D6. Clin Pharmacol Ther. 2020;107:154-70. https://doi.org/10.1002/cpt.1643.
5. Gaedigk A, Simon SD, Pearce RE, Bradford LD, Kennedy MJ, Leeder JS. The CYP2D6 activity score: translating genotype information into a qualitative measure of phenotype. Clin Pharmacol Ther. 2008;83:234-42. https://doi.org/10.1038/sj.clpt. 6100406.

6. Gaedigk A, Sangkuhl K, Whirl-Carrillo M, Klein T, Leeder JS. Prediction of CYP2D6 phenotype from genotype across world populations. Genet Med. 2017;19:69-76. https://doi.org/10.1038/ gim.2016.80.

7. Caudle KE, Sangkuhl K, Whirl-Carrillo M, Swen JJ, Haidar CE, Klein TE, et al. Standardizing CYP2D6 genotype to phenotype translation: consensus recommendations from the clinical pharmacogenetics implementation consortium and dutch pharmacogenetics working group. Clin Transl Sci. 2020;13:116-24. https://doi.org/10. $1111 /$ cts. 12692

8. Nofziger C, Paulmichl M. Accurately genotyping CYP2D6: not for the faint of heart. Pharmacogenomics. 2018;19:999-1002. https://doi.org/10.2217/pgs-2018-0105.

9. Yang Y, Botton MR, Scott ER, Scott SA. Sequencing the CYP2D6 gene: from variant allele discovery to clinical pharmacogenetic testing. Pharmacogenomics. 2017;18:673-85. https:// doi.org/10.2217/pgs-2017-0033.

10. Gaedigk A. Complexities of CYP2D6 gene analysis and interpretation. Int Rev Psychiatry Abingdon Engl. 2013;25:534-53. https://doi.org/10.3109/09540261.2013.825581.

11. Dalton R, Lee S-B, Claw KG, Prasad B, Phillips BR, Shen DD, et al. Interrogation of $C Y P 2 D 6$ structural variant alleles improves the correlation between $C Y P 2 D 6$ genotype and CYP2D6-mediated metabolic activity. Clin Transl Sci. 2020;13:147-56. https://doi. org/10.1111/cts.12695.

12. Pratt VM, Everts RE, Aggarwal P, Beyer BN, Broeckel U, Epstein-Baak R, et al. Characterization of 137 genomic DNA reference materials for 28 pharmacogenetic genes: a GeT-RM collaborative project. J Mol Diagn. 2016;18:109-23. https://doi. org/10.1016/j.jmoldx.2015.08.005.

13. Bousman CA, Jaksa P, Pantelis C. Systematic evaluation of commercial pharmacogenetic testing in psychiatry: a focus on CYP2D6 and CYP2C19 allele coverage and results reporting. Pharmacogenet Genom. 2017;27:387-93. https://doi.org/10.1097/ FPC. 0000000000000303 .

14. Ashley EA. The precision medicine initiative: a new national effort. JAMA. 2015;313:2119-20. https://doi.org/10.1001/jama. 2015.3595.

15. The Genome of the Netherlands Consortium, Francioli LC, Menelaou A, Pulit SL, van Dijk F, Palamara PF, et al. Wholegenome sequence variation, population structure and demographic history of the Dutch population. Nat Genet. 2014;46:818-25. https://doi.org/10.1038/ng.3021.

16. Turnbull C, Scott RH, Thomas E, Jones L, Murugaesu N, Pretty FB, et al. The 100000 Genomes Project: bringing whole genome sequencing to the NHS. BMJ. 2018;361:k1687. https://doi.org/10. 1136/bmj.k1687.

17. Numanagić I, Malikić S, Pratt VM, Skaar TC, Flockhart DA, Sahinalp SC. Cypiripi: exact genotyping of CYP2D6 using highthroughput sequencing data. Bioinformatics. 2015;31:i27-34. https://doi.org/10.1093/bioinformatics/btv232.

18. Twist GP, Gaedigk A, Miller NA, Farrow EG, Willig LK, Dinwiddie DL, et al. Constellation: a tool for rapid, automated phenotype assignment of a highly polymorphic pharmacogene, CYP2D6, from whole-genome sequences. NPJ Genom Med. 2016;1:15007. https://doi.org/10.1038/npjgenmed.2015.7.

19. Numanagić I, Malikić S, Ford M, Qin X, Toji L, Radovich M, et al. Allelic decomposition and exact genotyping of highly polymorphic and structurally variant genes. Nat Commun. 2018;9:1-11. https://doi.org/10.1038/s41467-018-03273-1. 
20. Lee S, Wheeler MM, Patterson K, McGee S, Dalton R, Woodahl EL, et al. Stargazer: a software tool for calling star alleles from next-generation sequencing data using $C Y P 2 D 6$ as a model. Genet Med. 2019;21:361. https://doi.org/10.1038/s41436-018-0054-0.

21. Lee S-B, Wheeler MM, Thummel KE, Nickerson DA. Calling star alleles With Stargazer in 28 pharmacogenes with whole genome sequences. Clin Pharmacol Ther. 2019;106:1328-37. https://doi. org/10.1002/cpt.1552.

22. Gordon AS, Fulton RS, Qin X, Mardis ER, Nickerson DA, Scherer S. PGRNseq: a targeted capture sequencing panel for pharmacogenetic research and implementation. Pharmacogenet Genom. 2016;26:161-8. https://doi.org/10.1097/FPC.0000000000000202.

23. Caspar SM, Schneider T, Meienberg J, Matyas G. Added value of clinical sequencing: WGS-Based profiling of pharmacogenes. Int J Mol Sci. 2020;21. https://doi.org/10.3390/ijms21072308.

24. Twesigomwe D, Wright GEB, Drögemöller BI, da Rocha J, Lombard Z, Hazelhurst S. A systematic comparison of pharmacogene star allele calling bioinformatics algorithms: a focus on CYP2D6 genotyping. Npj Genom Med. 2020;5:1-11. https://doi. org/10.1038/s41525-020-0135-2.

25. Gaedigk A, Turner A, Everts RE, Scott SA, Aggarwal P, Broeckel $\mathrm{U}$, et al. Characterization of reference materials for genetic testing of CYP2D6 alleles: a GeT-RM collaborative project. J Mol Diagn. 2019. https://doi.org/10.1016/j.jmoldx.2019.06.007.

26. Qiao W, Yang Y, Sebra R, Mendiratta G, Gaedigk A, Desnick RJ, et al. Long-read single molecule real-time full gene sequencing of cytochrome P450-2D6. Hum Mutat. 2016;37:315-23. https://doi. org/10.1002/humu.22936.

27. The 1000 Genomes Project Consortium. A global reference for human genetic variation. Nature. 2015;526:68-74. https://doi.org/ 10.1038/nature15393.

28. Raczy C, Petrovski R, Saunders CT, Chorny I, Kruglyak S, Margulies EH, et al. Isaac: ultra-fast whole-genome secondary analysis on Illumina sequencing platforms. Bioinformatics. 2013;29: 2041-3. https://doi.org/10.1093/bioinformatics/btt314.

29. Chen X, Sanchis-Juan A, French CE, Connell AJ, Delon I, Kingsbury $\mathrm{Z}$, et al. Spinal muscular atrophy diagnosis and carrier screening from genome sequencing data. Genet Med. 2020;18:1-9. https://doi.org/ 10.1038/s41436-020-0754-0.

30. Whirl-Carrillo M, McDonagh EM, Hebert JM, Gong L, Sangkuhl $\mathrm{K}$, Thorn $\mathrm{CF}$, et al. Pharmacogenomics knowledge for personalized medicine. Clin Pharmacol Ther 2012;92:414-7. https://doi. org/10.1038/clpt.2012.96.

31. Hosono N, Kato M, Kiyotani K, Mushiroda T, Takata S, Sato H, et al. CYP2D6 genotyping for functional-gene dosage analysis by allele copy number detection. Clin Chem. 2009;55:1546-54. https://doi.org/10.1373/clinchem.2009.123620.
32. Kiyotani K, Shimizu M, Kumai T, Kamataki T, Kobayashi S, Yamazaki H. Limited effects of frequent CYP2D6*36-*10 tandem duplication allele on in vivo dextromethorphan metabolism in a Japanese population. Eur J Clin Pharmacol. 2010;66:1065-8. https://doi.org/10.1007/s00228-010-0876-4.

33. Kim J, Lee S-Y, Lee K-A. Copy number variation and gene rearrangements in CYP2D6 genotyping using multiplex ligationdependent probe amplification in Koreans. Pharmacogenomics. 2012;13:963-73. https://doi.org/10.2217/pgs.12.58.

34. Qiao W, Martis S, Mendiratta G, Shi L, Botton MR, Yang Y, et al. Integrated CYP2D6 interrogation for multiethnic copy number and tandem allele detection. Pharmacogenomics. 2019;20:9-20. https://doi.org/10.2217/pgs-2018-0135.

35. Del Tredici AL, Malhotra A, Dedek M, Espin F, Roach D, Zhu G, et al. Frequency of $C Y P 2 D 6$ Alleles Including Structural Variants in the United States. Front Pharmacol. 2018;9. https://doi.org/10. 3389/fphar.2018.00305.

36. Chan W, Li MS, Sundaram SK, Tomlinson B, Cheung PY, Tzang CH. CYP2D6 allele frequencies, copy number variants, and tandems in the population of Hong Kong. J Clin Lab Anal. 2019;33: e22634. https://doi.org/10.1002/jcla.22634.

37. Black JL, Walker DL, O'Kane DJ, Harmandayan M. Frequency of undetected CYP2D6 hybrid genes in clinical samples: impact on phenotype prediction. Drug Metab Dispos. 2012;40:111-9. https://doi.org/10.1124/dmd.111.040832.

38. Gaedigk A, Twist GP, Leeder JS. CYP2D6, SULT1A1 and UGT2B17 copy number variation: quantitative detection by multiplex PCR. Pharmacogenomics. 2012;13:91-111. https://doi.org/ 10.2217/pgs.11.135.

39. Cai W-M, Nikoloff DM, Pan R-M, de Leon J, Fanti P, Fairchild M, et al. CYP2D6 genetic variation in healthy adults and psychiatric African-American subjects: implications for clinical practice and genetic testing. Pharmacogenomics J. 2006;6:343-50. https://doi. org/10.1038/sj.tpj.6500378.

40. Salyakina D, Roy S, Wang W, Oliva M, Akhouri R, Sotto I, et al. Results and challenges of Cytochrome P450 2D6 (CYP2D6) testing in an ethnically diverse South Florida population. Mol Genet Genomic Med. 2019;7. https://doi.org/10. $1002 / \mathrm{mgg} 3.922$.

41. Dolzhenko E, van Vugt JJFA, Shaw RJ, Bekritsky MA, van Blitterswijk M, Narzisi G, et al. Detection of long repeat expansions from PCR-free whole-genome sequence data. Genome Res. 2017;27:1895-903. https://doi.org/10.1101/gr.225672.117.

42. Spies N, Zook JM, Salit M, Sidow A. svviz: a read viewer for validating structural variants. Bioinformatics. 2015;31:3994-6. https://doi.org/10.1093/bioinformatics/btv478. 